\title{
Asientos (1995) de François L. Woukoache
}

\section{(2) OpenEdition}

\section{Journals}

Édition électronique

URL : https://journals.openedition.org/recherchestravaux/697

DOI : 10.4000/recherchestravaux.697

ISSN : 1969-6434

\section{Éditeur}

UGA Éditions/Université Grenoble Alpes

\section{Édition imprimée}

Date de publication : 1 novembre 2014

Pagination : 201-213

ISBN : 978-2-84310-282-0

ISSN : 0151-1874

Référence électronique

"Asientos (1995) de François L. Woukoache », Recherches \& Travaux [En ligne], 84 | 2014, mis en ligne le 01 avril 2016, consulté le 29 octobre 2021. URL : http://journals.openedition.org/recherchestravaux/ 697 ; DOI : https://doi.org/10.4000/recherchestravaux.697 


\section{Asientos (1995) de François L. Woukoache}

\section{Introduction \\ (Didier Coureau)}

François L. Woukoache est un cinéaste né au Cameroun en 1966, auteur de plus d'une vingtaine de films. Après des études supérieures scientifiques suivies en France, puis des études de cinéma en Belgique à l'INSAS, il s'est installé au Rwanda à la fin des années I990, après que le génocide des Tutsis a été perpétré par les Hutus. Il mène depuis lors un travail de réflexion et de mémoire, à travers la réalisation de films (Nous ne sommes plus morts; Humura; Icyizere, l'espoir...), la mise en place d'actions de formation en cinéma à l'université nationale du Rwanda - où il a supervisé la réalisation des deux premiers courts métrages de fiction du pays - et d'archivage audiovisuel des témoignages (mémoire du génocide et réconciliation).

Pour le présent volume, il a accepté de confier le texte de son film Asientos (I995), écrit par Claire Jaumain et par lui-même. Film-réflexion sur la traite des Noirs, film-essai, mais aussi film-poème qui croise en son cœur même la poésie d'Aimé Césaire dans son Cahier d'un retour au pays natal, et où plusieurs voix off (prononcées par Fabienne Loriaux, Sidiki Bakaba et N'Goné Fall) s'entretissent. L'image, au format $35 \mathrm{~mm}$, est l'œuvre du chef opérateur Raymond Fromont (qui a souvent collaboré avec Chantal Akerman), et le son d'Antoine Bonfanti (qui travailla entre autres avec Chris Marker et Jean-Luc Godard).

Le texte des voix off d'Asientos est ici précédé par le texte de présentation du film, écrit par François L. Woukoache, et suivi d'un poème dédié à son film. 


\section{Texte de présentation d'Asientos (François L. Woukoache)}

Cent ans se sont écoulés depuis qu'a pris fin la déportation d'Africains vers le Nouveau Monde.

Le cinéma fête ses cent ans.

Faire un film sur la traite des Noirs c'est se confronter à l'absence d'images (il n'y a pas d'images d'archives ni de témoins vivants).

Asientos est traversé par une série d'interrogations :

Comment dire une souffrance qui a eu lieu il y a plus d'un siècle alors qu'on a déjà tant de mal à faire accepter celle d'il y a cinquante ans, et que celles d'aujourd'hui semblent laisser indifférent? Comment dire que c'est une histoire d'hommes, donc faite de souffrances et de larmes?

Comment dire cette mémoire sans témoins et dont les lieux subissent les assauts terribles du temps?

Peut-on imaginer aujourd'hui les horreurs des siècles passés?

Quelles images?

Le film raconte l'histoire d'un jeune africain traumatisé par la violence qu'il perçoit dans le monde.

Pour échapper à cette violence, il se réfugie dans son imaginaire et se trouve confronté à un pan de l'Histoire du peuple noir : la traite.

Le film est un voyage à travers l'imaginaire, à travers la mémoire.

Voyage au cœur de la douleur et de la souffrance, voyage au cœur de l'horreur.

Questionner inlassablement les lieux vides.

Lieux vides et remplis de mémoire.

Filmer pour la Mémoire, contre l'oubli.

Filmer pour lutter contre l'amnésie sélective qui permet d'éviter de se confronter aux moments de faillite de la raison humaine à l'origine des pages les plus horribles de l'Histoire.

Essayer de franchir la barrière du temps.

Le temps qui travaille.

Le travail du temps sur la matière : 
Les pierres, l'eau, le sable, le vent, le ciel... les corps.

Matières opaques, indéchiffrables, muettes, abstraites.

Le temps des Hommes, le temps du Monde... et le temps du film.

Moins d'une heure de film pour :

- lever le manteau de silence qui sépare ces événements de l'espace social contemporain.

- exprimer à la fois la souffrance et la difficulté de dire cette souffrance.

- faire le deuil de ce «moment historique».

Travail de deuil nécessaire pour enfin entrer définitivement dans la modernité. 


\section{Texte du film Asientos \\ (François L. Woukoache et Claire Jaumain)}

\section{Prologue}

Asiento signifie la chose assise, convenue.

Au Xvi ${ }^{\mathrm{e}}$ siècle, l'État espagnol confie aux nouvelles puissances que sont les compagnies financières et commerciales, l'organisation de la Traite des Noirs, sous forme d'asientos ou licences.

L'asiento est un contrat de droit public entre le roi et un contractant.

C'est une concession unilatérale accordée par le roi pour la livraison d'un nombre précis de Nègres aux colonies, moyennant certaines prestations

Le premier asiento de Nègres, en I528, fut accordé à des Allemands, Eynger et Sayler, gens de la maison de Charles Quint. Ils recevaient le monopole de quatre mille Noirs à livrer en quatre ans, à quarante ducats par tête, moyennant vingt mille ducats à payer au trésor.

Ils vendirent les licences aux Portugais.

\section{Elle (voix)}

La première fois que nous nous sommes rencontrés...

Je ne comprends pas ce qu'il veut. Je n'ai jamais été en Afrique.

Je ne sais pas à quoi ressemblent les terres ni les peuples d'Afrique.

Je l'écoute parler...

Trop d'images, ici en Europe dit-il. Trop d'images, impuissantes.

L'absence d'images là-bas. Et la difficulté à remplir la case vide de sa mémoire. Lui il a étudié en France, mais son corps est resté là-bas. Son corps est là-bas.

Son corps est traversé par la souffrance de ne pas avoir accès à ce passé qui le terrifie.

Là-bas dit-il, quand on vit une chose dure, on n'en parle pas. Les choses graves restent silencieuses.

Il a besoin de se souvenir. Mais comment se souvenir de ce qui n'existe nulle part?

Comment dire : voilà! Cela a eu lieu, et jamais plus.

Il a peur. Il a peur que cela recommence ailleurs, ici, maintenant c'est en train de recommencer.

Pourquoi vouloir encore fouiller le passé?

Des enfants naissent, des enfants jouent, cela ne suffit-il pas? 
J'ai accepté de l'aider à remplir la case vide de sa mémoire.

À mettre un nom sur les âmes mortes des défunts.

Là où je souffre dit-il, il n'y a rien pour souffrir.

Rien non plus pour supporter cette souffrance.

Comment atteindre le lieu de cette souffrance?

Comment savoir ce qui s'est passé?

Il n'y a rien à montrer, il n'y a rien.

\section{Première partie}

\section{Voix $I$}

Que de sang dans ma mémoire!

Dans ma mémoire sont des lagunes.

Elles sont couvertes de têtes de morts.

Elles ne sont pas couvertes de nénuphars...

... Dans ma mémoire sont des lagunes.

Sur leurs rives ne sont pas étendus des pagnes de femmes.

Ma mémoire est entourée de sang.

Ma mémoire a sa ceinture de cadavres ${ }^{\text {! }}$

\section{Voix $I$}

Mon corps s'est refermé sur ses blessures.

Mon corps doit soulever tant de siècles.

Ces siècles qui nous séparent de toi.

Depuis des siècles, mes frères, tes ancêtres crient. Et personne n'entend.

Ils ont disparu dans l'histoire.

Tu ne peux pas y croire. Tu ne veux pas y croire.

$\mathrm{Tu}$ as peur... Tu avales les morceaux de ton enfance qui ne te protège plus. Écoute...

En 1492 Christophe Colomb arrive en Amérique.

Après les Espagnols, les Français et les Anglais occupent peu à peu toutes les îles du continent américain.

En 1492 la population Indienne est d'environ six millions d'individus, vers I900, les Indiens ne sont plus que deux cent cinquante mille.

I. A. Césaire, Cahier d’un retour au pays natal, Paris, Présence africaine, coll. «Poésie», 1995, p. 35 . 
L'extermination des Indiens va provoquer la déportation de millions d'Africains comme main d'œuvre dans les plantations de canne à sucre et de coton en Amérique.

Du nord au sud et de l'est à l'ouest, depuis les ports de l'Atlantique et d'ailleurs, le vieux continent arme ses bateaux pour de sinistres trafics.

Jamais auparavant les Européens ne s'étaient lancés dans la chasse aux esclaves sur une échelle aussi gigantesque.

C'est une chasse systématique, entreprise dans la seule intention de se procurer des esclaves.

Les bons bourgeois de Bordeaux, Bristol, Copenhague ou Lisbonne investissent dans ce commerce et tiennent à être appelés négociants et non marchands. Car vois-tu, ils font du gros et non du détail.

\section{Voix $I$}

Notre-Dame-de-l'Espérance, Notre-Dame-de-la-Pitié, le Duc de Bourgogne...

Voix 2 (chevauche sur la suite de Voix I)

...Notre-Dame-de-l'Espérance, Notre-Dame-de-la-Pitié, le Duc de Bourgogne, Jésus-Marie-Joseph, Saint Charles, Deux soeurs, Fille Unique, la Ninette, L'Andromaque, Belle Pauline, le Contrat Social.

\section{Voix I (suite)}

Rien ne distingue les bateaux de traite des autres bateaux de commerce, parce que les négriers utilisent des voiliers déjà fatigués par des années de transport.

\section{Voix I (suite, chevauche sur la fin de Voix 2)}

Jésus-Marie-Joseph, Saint Charles, Deux sœurs, Fille Unique...

Le commerce des esclaves noirs a duré quatre siècles.

Pendant quatre siècles, l'Afrique est vidée de ses enfants les plus robustes

\section{Voix 2}

C'est une saignée démographique sans précédent dans l'histoire de l'humanité.

La carte politique de l'Afrique subit de profondes modifications.

Voix I (chevauche sur la suite de Voix 2)

Au Djolof, au Cayor, au Songhay, au Congo, à Kaarta, à Ségou, en Abomey, en Ashanti...

Voix 2 (suite)

Les pouvoirs traditionnels sont déstabilisés. 
Les bénéfices du commerce négrier sont plus importants que ceux obtenus avec le commerce du kola, de livoire ou du bétail.

L'ambition et l'appât du gain dressent les chefs les uns contre les autres.

Les guerres se multiplient. Les populations sont capturées et vendues.

Pendant quatre siècles, les pillages, les vols, les viols, les rafles de personnes et du bétail...

\section{Voix $I$}

La destruction des valeurs morales et spirituelles...

Voix 2 (suite)

L'équilibre social est brisé.

La pression de la demande européenne en esclaves brise l'équilibre social.

\section{Voix $I$}

Tous les jours je suis venu ici.

Tous les jours je t'ai attendu.

J'ai voulu te parler.

Où étais-tu?

Tu ne regardes pas, tu n'écoutes pas. Tu joues.

Tu es jeune, c'est comme si tu n'étais pas né.

Tu ne sais même pas que j'ai existé.

Que veux-tu?

Laisse-moi, tu n'auras rien de moi.

Je suis mort, tu ne peux entrer dans la mort.

\section{Deuxième partie}

\section{Voix $I$}

Tes ancêtres, mes frères?

Je les vois... Leurs membres sont sectionnés, leurs yeux aveuglés.

Mes frères...

Je les vois comme dans un naufrage sortir lentement des ténèbres.

Leur peau est un linceul, leur corps une enveloppe de silence.

Oui, je les vois.

Ils sont là!

Simples marchandises transportant d'autres marchandises

Dans des caravanes qui avancent à coups de fouet le long des pistes. 
Les hommes sont enchaînés par groupe de dix ou vingt

Ils portent autour du cou des carcans de fer reliés par une chaîne cadenassée Les récalcitrants ont les mains attachées derrière le dos et un morceau de bois en travers de la bouche.

Parfois, pour permettre à la mère de porter l'ivoire, qui coûte plus cher qu'un enfant en bas âge, on se débarrasse de l'enfant en le transperçant d'un coup de lance ou en lui broyant le crâne contre un arbre.

Oui je les vois...

... Captifs regroupés près des côtes...dans des esclaveries... attendant d'être vendus

Toi qui as grandi sans savoir

Toi qui as lu tant de livres

Qui as vu et comparé tant de chiffres

Toi qui as retracé les itinéraires des bateaux négriers

Tu ne sais toujours pas pourquoi la mer ne rejette pas ces cadavres

Écoute...

Tu peux entrer dans l'histoire mais tu ne peux en sortir

Tu ne peux pas déterrer les morts, ni leurs cris, ni leurs révoltes, ni leurs blessures Je suis mort et tu n'étais pas né.

Je mourrai sans t'avoir rien laissé

Orphelin de ceux qui sont partis, pas revenus.

Tes ancêtres, Mes frères.

Tu veux visiter...

VOIR?

Va.

Regarde.

Tu verras des murs, des pierres posées les unes sur les autres.

Tu verras le vent et la mer.

Le sable que tu piétines et qui te réchauffe les pieds.

C'est un marché.

Comme dans tout marché, il faut savoir choisir.

Le chirurgien, procède à la visite corporelle :

Surtout pas de vieux à peaux ridée, testicules pendants et ratatinés, tondu et rasé.

Les grands nègres efflanqués, ventre flasque, air imbécile se vendent mal.

Pour les femmes, ni tétons cabris sans seins, ni mamelles pendantes et flasques. Ce qu'on apprécie surtout est une bonne reproductrice. 
Le développement du bassin et de la poitrine est particulièrement estimé Les femmes doivent avoir «les seins debout».

Un jeune homme d'une vingtaine d'années à la dentition saine, musclé et un peu gras, est une "pièce d'Inde", il se vend très cher.

Le manque d'une seule dent, une simple tâche dans l'œil rend un esclave défectueux comme esclave de première qualité.

J'étais nu, sans paroles, livré aux envahisseurs.

Mon corps s'est perdu dans un naufrage.

J'ai rejoint mes frères entassés sans noms dans les fêlures de l'histoire.

Je n'existe plus.

Fais quelque chose pour moi :

SOUVIENS-TOI!

Souviens-toi des affres de mes frères, tes ancêtres...

Souviens-toi des angoisses et des mensonges.

De la solitude et du froid.

De la violence et de la cruauté.

Souviens-toi des langues coupées, des jarrets tranchés, des lances d'aciers traversant les poitrines.

Souviens-toi de l'odeur de la mort!

\section{Troisième partie}

\section{Voix 2}

Les captifs sont répartis par lots.

Marqués au fer. Tondus.

Être marqué au fer inscrit à jamais la condition d'esclave dans la chair.

Moins de trente kilos, le captif est déclaré «inapte temporaire» il est mis dans une cellule spéciale afin d'être engraissé.

Si un captif refuse de manger, on lui ouvre la bouche avec un appareil spécial :

Le speculum oris

Le speculum oris est en vente dans les magasins de Nantes et de Liverpool.

Des hommes et des femmes meurent.

Les épidémies font des ravages.

Les épidémies de dysenterie et de fièvre jaune.

Le scorbut et la variole tuent.

Des enfants meurent. 
Les épidémies font des ravages.

Les épidémies de dysenterie et de fièvre jaune.

Des hommes et des femmes meurent.

Le scorbut et la variole tuent.

Les épidémies font des ravages.

Des enfants meurent.

Les épidémies de dysenterie et de fièvre jaune.

Marqués au fer. Tondus.

Être marqué au fer inscrit à jamais la condition d'esclave dans la chair.

Toute la nuit elle a porté le deuil.

Le deuil des millions de morts sans sépultures.

Les morts d'hier, et ceux d'aujourd'hui.

Toute la nuit elle a crié des noms.

Des noms à jamais perdus dans les fêlures de l'histoire.

Écoutez...

Un chien aboie

Un enfant pleure

Le jour devient la nuit...

C'est la danse des vautours et des charognards.

Quelqu'un pleure,

Quelqu'un traverse l'océan.

Dix millions de personnes traversent l'océan

Peut-être Vingt? Ou cinquante? Personne ne sait exactement

Et TOMBA?

Qui se souvient de TOMBA?

Il fomenta une révolte sur un bateau avec une femme pour complice.

Tomba fut tué.

Que l'on mange son cœur et son foie ordonna le commandant a deux de ses compagnons.

Qu'on la pende par les pouces.

Qu'on la fouette.

Qu'on la taillade à coups de couteaux jusqu'à ce qu'elle meure.

Tomba le Mandjak

Qui se souvient de toi?

Peut-être étais-tu Yorouba ou Bambara? Personne ne le sait.

Et la femme?

Était-elle Bororo ou Peul? Peut-être Toucouleur?

Comment savoir? Qui nous le dira?Les âmes mortes restent silencieuses. 


\section{Voix 2}

La terre a brûlé.

La terre est abreuvée de sang.

La terre est baignée de larmes.

Les gestes et les mots n'ont plus de sens,

Le silence a recouvert les chansons et les danses.

Quelqu'un pleure, quelqu'un souffre.

C'est la souffrance de celui qui n'a pas de nom.

Ce sont les larmes d'un peuple qui n'a pas de tombes.

\section{Voix I}

Que de sang dans ma mémoire!

Dans ma mémoire sont des lagunes.

Elles sont couvertes de têtes de morts.

Elles ne sont pas couvertes de nénuphars...

... Dans ma mémoire sont des lagunes.

Sur leurs rives ne sont pas étendus

des pagnes de femmes.

Ma mémoire est entourée de sang.

Ma mémoire a sa ceinture de cadavres² !

\section{Épilogue}

\section{Elle (voix)}

Nous nous sommes tenus à distance de l'horreur, refusant d'assumer nos crimes.

Il est si facile de croire que cela n'a pas eu lieu.

Cette époque fabrique l'oubli.

Je l'écoute parler...

Le temps efface dit-il, le temps rejette dans l'ombre.

Le temps ne tiens pas compte de nos malheurs...

Mais ces corps...

Comment les oublier?

Ces corps perdus à la peau trouée. Ces corps déchiquetés...

Comment oublier alors qu'aujourd'hui encore, ce peuple marche vers sa mort?

Que ses images alimentent un commerce dont nous sommes les clients?

2. A. Césaire, Cahier d'un retour au pays natal, ouvr. cité. 
Ce peuple est noir. Comme moi.

Oui, je sais. Tu es blanche me dit-il.

Je me demande...

Comment l'amour peut disparaître,

pourquoi cette indifférence qui tue.

Plus cruellement.

Je regarde ce siècle qui se déchire,

L'histoire qui se répète et j'ai peur...

Je voudrais m'approcher...

Je voudrais m'approcher, m'asseoir... Me taire...

Oui... Se taire dit-il

Écouter le silence.

Réapprendre à regarder,

pour voir l'innommable.

Un jour, peut-être. 


\title{
Temps tissage \\ (Asientos) $^{3}$
}

\author{
Océan \\ écran bleu \\ entre deux continents \\ Plis \\ les vagues \\ le tissu déchirure \\ la peau crevassée \\ cartographie \\ les rides les âmes \\ enracinées \\ au cour du sable \\ Océan \\ linceul bleu \\ aux cargaisons \\ d'absence \\ Océan \\ mémoire infranchissable \\ Une femme marche \\ bord blanc du rivage \\ Un fil sort des eaux \\ presque invisible \\ le temps.
}

\section{Didier Coureau}

3. Ce poème a été publié une première fois dans la revue Friches (Cahiers de poésie verte), n 66, St-Yrieix, printemps 1999, p. 26. 